great importance for the study of nuclear structure. It is also possible that the discrepancy would be reduced or even eliminated by a full relativistic analysis, but this has not yet been done.

\section{Sun-shuttling lizards}

from our Animal Ecology Correspondent

LIZARDS are distinctive by their relative absence from temperate forests. This situation, on the face of it, seems anomalous in view of the great abundance of insect prey species in this sort of habitat. Huey (Science, 184, 1001 ; 1974) now points out that this anomaly may be resolved by considering the ecological difficulties encountered by poikilothermic carnivores.

Unable to thermoregulate physiologically, most lizards move about from sunshine to shade or modify their body posture to alter surface areas exposed to heat sources and thus thermoregulate behaviourally (Cowles and Bogert, Bull. Am. Mus. nat. Hist., 83, 261; 1944; Heath, Univ. Calif. Publs Zool., 64, 97; 1965). Their thermal strategy can be described as heliothermy. It is theoretically possible to predict the amount of behavioural thermoregulation that would maximise net profits by calculating the 'costs' of a physical movement into or out of sunshine as against the 'gain' involved in the attainment and maintenance of a specific body temperature. In Huey's study on Anolis cristatellus in Puerto Rica the energetic cost of physical movement from shade into sunshine was estimated by measuring the shortest practicable distance from a perch in the shade to one in the sunshine. Anoles occupy a variety of habitats. Huey chose to study two adjacent populations, one in a shaded forest and the other in an open meadowland. If a tree or other perch was totally shaded and offered no sunlit patches, this fact was noted. Many more lizards in the forest were unable to reach full sun without changing perches (which usually meant changing trees) than were those in the meadow. Furthermore, those lizards in the forest that could reach the sunshine were significantly further from it than were those in the open. Thus there seemed little doubt that the energetic 'cost' of heliotactic thermoregulation was far higher in the forest population.

During the early morning the cloacal temperature of anoles in the forest increased significantly slower than that of meadow lizards and was strongly correlated with ambient air temperature. Such a strong relation did not exist between lizards living in the open and the surrounding air; body temperature of lizards in the open fluctuated by only $1.6^{\circ} \mathrm{C}$ during the day as compared with $4.9^{\circ} \mathrm{C}$ in the forest.
The interpretation of these observations is that behavioural thermoregulation occurs only where sun and shade conditions are juxtaposed. Where 'costs' are high, as in the three-dimensional environment of the forest, anoles are passive to ambient conditions. Under these conditions the rewards of sun-shuttling are insufficient and this is reflected in very low population densities. What is the evolutionary significance of this exploitational strategy?

A. cristatellus is a remarkable reptile in having such a plastic ecology. The genus is known for its ability to colonise islands de novo as well as to form sympatric species on islands already colonised (Williams, Q. Rev. Biol., 44, $345 ; 1969$ ), and its degree of thermal tolerance possibly accounts for its widespread success in the Caribbean. Those populations of $A$. cristatellus that occupy open habitats have an energy budget from which reproduction can have a larger share than is possible in forest populations. Although lizards in the two habitats are indistinguishable in terms of dewlap colour, snout-vent length or midbody scale rows, and do not differ with respect to preferred body temperature in a laboratory gradient, it would seem likely that their nopulations are in the process of active divergence. Perhaps sun-shuttling is the key to two questions: first, the absence of lizards in forests; and second, the complexity of Anolis speciation.

\section{Magnetospheres of Earth and Jupiter}

from N. A. Heard and A. R. L. Tatnall

THE tragic death of Neil Brice earlier this year in an air crash over the Pacific led to the renaming of the symposium in Frascati (May 28-31) on the magnetospheres of Earth and Jupiter, the Neil Brice Memorial Symposium. After V. Formisano (Spazio Plasma Laboratory, Frascati) had opened the meeting, C. Kennel (University of California) described the valuable contribution of Neil Brice to the study of the Earth's magnetosphere.

The symposium fell naturally into two parts. In the first half the Earth's magnetosphere was reviewed and results from several satellites were presented. K. Schindler (Ruhr University, Bochum) and V. Vasylinnas (Massachusetts Institute of Technology) presented theoretical calculations based on models of the magnetosphere; some attempt was made to extend the model of the Earth's magnetosphere to that of Jupiter.

It has been known for some time that Jupiter is a very intense planetary radio source but, as D. Gurnett (University of Iowa) showed, the Earth is not to be outdone in this respect. Imp- 6 and 8 satellites have revealed that the Earth is a comparable radio source and in the frequency range from $50 \mathrm{kHz}$ to 500 $\mathrm{kHz}$, at peak intensity, the total power emitted is about $10^{9} \mathrm{~W}$. This radio emission is associated with discrete auroral arcs and seems to originate from altitudes of about $2,000 \mathrm{~km}$. It was suggested that a satellite at this altitude might provide some interesting results.

As R. W. Fredericks (TRW Systems, California) pointed out the value of studies of wave-particle interactions to the understanding of the Earth's magnetospheric processes; it was generally agreed that similar studies of the wave-particle interactions in Jupiter's magnetosphere are essential.

Motion of the Earth's bow shockthe shock caused by the supersonic solar wind impinging on the Earth's magnetic field-was discussed by several speakers. The causes of shock motion are not understood but, from data from Heos 1, Formisano and G. Mastrantonio (Frascati) showed that a satellite is likely to encounter the bow shock in motion if tangential discontinuities are the main cause of motion.

E. J. Smith (Jet Propulsion Laboratory, California) confirmed that the outer boundary of Jupiter's magnetosphere fluctuates considerably, a possibility mentioned by several speakers. The vector helium magnetometer results have revealed that the magnetopause is crossed twice on the inbound pass and a number of times on the outbound pass. An interesting feature of Jupiter's magnetic dipole field is that it is directed opposite to that of the Earth. This dipole field is inclined $15^{\circ}$ to the rotation axis and offset about $0.1 R_{J}$ north of the equatorial plane and about $0.2 R$.r towards longitude $170^{\circ}$. From this estimated dipole moment the surface field is between 2.3 and 11.7 gauss compared with the Earth's field of between 0.3 and 0.6 gauss. The dipole approximation for Jupiter is even less valid than in the case for Earth, because of the considerable contribution from current sheets around the planet. Pioneer 10 has also confirmed the presence of helium on Jupiter and the existence of an internal energy source in the planet because the excess radiation is 2 to $2.5 \times$ the solar input.

The role of the Galilean moons is still uncertain but, as several speakers showed, Io in particular seems to influence the particle flux considerably. Io is about the same size as Earth's moon and occultation experiments have shown that it has an ionosphere and a surface pressure of $10^{-6}$ bar.

The fly-by of a planet obviously leaves a number of unanswered questions and all speakers emphasised the difficulty in distinguishing between spatial and temporal variations. 